\title{
Thuja orientalis reduces airway inflammation in ovalbumin-induced allergic asthma
}

\author{
IN-SIK SHIN ${ }^{1,2}$, NA-RAE SHIN ${ }^{1}$, CHAN-MI JEON ${ }^{1}$, OK-KYOUNG KWON ${ }^{1}$, JU-MI HONG ${ }^{1}$, \\ HUI-SEONG KIM ${ }^{1}$, SEI-RYANG OH ${ }^{1}$ and KYUNG-SEOP AHN ${ }^{1}$
}

\author{
${ }^{1}$ Natural Medicine Research Center, Korea Research Institute of Bioscience and Biotechnology, Cheongwon-gun, \\ Chungbuk 363-883; ${ }^{2}$ College of Veterinary Medicine, Chonnam National University, Gwangju 500-757, Republic of Korea
}

Received January 14, 2014; Accepted July 31, 2014

DOI: $10.3892 / \mathrm{mmr} .2015 .3910$

\begin{abstract}
Thuja orientalis (TO) may be used as a herbal remedy for the treatment of numerous inflammatory diseases. In the present study, the effects of TO were evaluated on airway inflammation in ovalbumin (OVA)-induced allergic asthma and RAW264.7 murine macrophage cells. The effects of TO on the production of proinflammatory mediators, were determined in RAW264.7 cells that had been stimulated with lipopolysaccharide (LPS). Furthermore, an in vivo experiment was performed on mice that were sensitized to OVA and then received an OVA airway challenge. TO was administered by daily oral gavage at a dose of $30 \mathrm{mg} / \mathrm{kg}, 21-23$ days after the initial OVA sensitization. TO was shown to reduce nitric oxide production and reduce the relative mRNA expression levels of inducible nitric oxide synthase (iNOS), interleukin (IL)-6, cyclooxygenase-2, matrix metalloproteinase (MMP)-9, and tumor necrosis factor- $\alpha$ in RAW264.7 cells stimulated with LPS. In addition, TO markedly decreased the inflammatory cell counts in bronchial alveolar lavage fluid, reduced the levels of IL-4, IL-5, IL-13, eotaxin and immunoglobulin E, and reduced airway hyperresponsivenes, in the OVA sensitized mice. Furthermore, TO attenuated airway inflammation and mucus hypersecretion, induced by the OVA challenge of the lung tissue. TO also reduced the expression of iNOS and MMP-9 in lung tissue. In conclusion, TO exerted anti-inflammatory effects in an OVA-induced allergic asthma model, and in LPS-stimulated RAW264.7 cells. These results suggest that TO may be a useful therapeutic agent for the treatment of inflammatory diseases, including allergic asthma.
\end{abstract}

Correspondence to: Dr Kyung-Seop Ahn, Natural Medicine Research Center, Korea Research Institute of Bioscience and Biotechnology, 30 Yeongudanji-ro, Ochang-eup, Cheongwon-gun, Chungbuk 363-883, Republic of Korea

E-mail: ksahn@kribb.re.kr

Key words: Thuja orientalis, inflammation, inducible nitric oxide synthase, matrix metalloproteinase-9, asthma

\section{Introduction}

Allergic asthma is a common inflammatory disease, which affects 300 million people worldwide (1). Allergic asthma results from exposure to allergens which may include pollens, house dust, animal dander, inhalants, foods and air pollutants (2). Patients with allergic asthma exhibit clinical features including wheezing, breathlessness, dyspnea and coughing (3). These symptoms result from airway inflammation, which is associated with the recruitment of inflammatory cells into the airway, mucus overproduction and airway hyperresponsiveness (2). Airway inflammation is a complex response that is associated with numerous factors including $\mathrm{T}$ helper (Th) type 2 cytokines, proinflammatory proteins, chemokines and growth factors (1). The importance of these mediators in the development of allergic asthma has been demonstrated in previous studies (2-4). Among these mediators, inducible nitric oxide synthase (iNOS) has been shown to have a pivotal role in the production of nitric oxide (NO), which acts as a powerful aggravator of allergic asthma (5). Asthmatic conditions are associated with an overexpression of iNOS in the airways, and significantly elevated NO production (5). Previous studies have demonstrated that NO activates proinflammatory signaling and Th2 responses in allergic asthma (6). Furthermore, the suppression of iNOS expression in the airways has been shown to reduce asthmatic responses in numerous asthma models (7).

Thuja orientalis (TO) is an evergreen tree, from which extracts are used in Korean traditional medicine. It is used to treat numerous diseases, including gout, rheumatism, diarrhea and chronic tracheitis, as demonstrated by numerous in vivo and in vitro studies (8-11). Previous studies have demonstrated that TO possesses antioxidant, anticancer, and anti-inflammatory properties (12-14). However, there are currently no studies on the effects of TO on asthmatic responses that include airway hyperresponsiveness (AHR), inflammation, and mucus hypersecretion.

The aims of the present study were to examine the effects of TO on airway inflammation in an ovalbumin (OVA)-induced allergic asthma murine model, by measuring Th2 cytokines, AHR, chemokines, immunoglobulin (Ig) E, and histologically analyzing lung tissue. To further investigate the possible anti-inflammatory mechanisms of TO, the expression levels 
of proinflammatory proteins, including iNOS, cyclooxygenase (COX)-2 and matrix metalloproteinase (MMP)-9, were determined in RAW264.7 cells and the allergic asthma murine model.

\section{Materials and methods}

Cell culture and viability. RAW264.7 murine macrophage cells were purchased from the American Type Culture Collection (Manassas, VA, USA). The cells were incubated at $37^{\circ} \mathrm{C}$ in $5 \% \mathrm{CO}_{2}$ in Dulbecco's modified Eagle's media (DMEM; Gibco-BRL, Carlsbad, CA, USA), supplemented with $100 \mathrm{U} / \mathrm{ml}$ penicillin, $100 \mu \mathrm{g} / \mathrm{ml}$ streptomycin and $5.5 \%$ fetal bovine serum (Gibco-BRL). The cells were also treated with $0.05 \%$ dimethyl sulfoxide as a vehicle control. The cells were seeded in 96-well plates at a density of $5 \times 10^{4}$ cells/well and incubated in serum-free medium in the presence of different concentrations of TO. Following a $24 \mathrm{~h}$ incubation, the cellular viability was determined using an MTT assay. TO was obtained from the Plant Extract Bank at the Korea Research Institute of Bioscience and Biotechnology (PB1411.1; Daejeon, South Korea). All experiments were performed in triplicate. TO concentrations were used that have been shown to be nontoxic in other biological systems, based on MTT results (Fig. 1A).

Measurement of NO production. The cells $\left(2.5 \times 10^{5}\right.$ cells $\left./ \mathrm{ml}\right)$ were seeded in 96-well plates in phenol red-free DMEM, and treated with different concentrations $(10,20,30$, and $40 \mu \mathrm{g} / \mathrm{ml})$ of TO for $1 \mathrm{~h}$, followed by an incubation in the presence of lipopolysaccharide (LPS; $1 \mu \mathrm{g} / \mathrm{ml}$ ) for $24 \mathrm{~h}$. Nitrite accumulation in the culture medium was measured using Griess reagent (Promega Corporation, Madison, WI, USA). The absorbance was measured at $535 \mathrm{~nm}$, using a microplate reader (Bio-Rad, Hercules, CA, USA).

Reverse transcription-polymerase chain reaction (RT-PCR). Total RNA was isolated from the cells using TRIzol ${ }^{\circledR}$ reagent (Invitrogen Life Technologies, Carlsbad, CA, USA). A total of $2 \mu \mathrm{g}$ of RNA was reverse transcribed, to synthesize single-stranded cDNA, using the commercially available kit (Qiagen, Valencia, CA, USA). The cDNA was then subjected to PCR. The primer sequences (Bioneer, Daejeon, Korea) used were as follows: tumor necrosis factor (TNF)- $\alpha$, sense, $5^{\prime}$-GTG GAA CTG GCA GAA GAG GC-3', antisense, 5'-AGA CAG AAG AGC GTG GTG GC-3'; COX-2, sense, 5'-CAA GTC TTT GGT CTG GTG CCT G-3', antisense, 5'-GTC TGC TGG TTT GGA ATA GTT GC-3'; iNOS, sense, 5'-CAA GAG TTT GAC CAG AGG ACC-3', antisense, 5'-TGG AAC CAC TCG TAC TTG GGA-3'; interleukin (IL)-6, sense, 5'-GAG GAT ACC ACT CCC AAC AGA CC-3', antisense, 5'-AAG TGC ATC ATC GTT GTT CAT ACA-3'; MMP-9, sense, 5'-AAG CAC ATG CAG AAT GAG TAC CG-3', antisense, 5'-GTG GGA CAG CTT CTG GTC GAT-3'; and $\beta$-actin, sense, 5'-CGC TCA TTG CCG ATA GTG AT-3'; and antisense 5'-TGT TTG AGA CCT TCA ACA CC-3'. The PCR products were fractionated and analyzed qualitatively by $1.5 \%$ agarose gel electrophoresis, stained with $5 \mu \mathrm{g} / \mathrm{ml}$ ethidium bromide for visualization.

Gelatin zymography. The cells $\left(2.5 \times 10^{5}\right.$ cells $\left./ \mathrm{ml}\right)$ were seeded into 96-well plates in phenol red-free DMEM and treated with different concentrations (10, 20,30 and $40 \mu \mathrm{g} / \mathrm{ml})$ of TO for $1 \mathrm{~h}$, followed by an incubation in the presence of LPS for $24 \mathrm{~h}$. The cell supernatants were collected and were loaded for gelatin zymography. SDS-PAGE zymography was performed according to the methods of Heussen and Dowdle (15), to determine gelatinase activities. Briefly, zymogram gels, consisting of $10 \%$ polyacrylamide gel containing SDS and $1 \%$ gelatin, were used as the MMP substrate. The gels were washed in $2.5 \%$ Triton X-100 for $1 \mathrm{~h}$ to remove the SDS, followed by an incubation at $37^{\circ} \mathrm{C}$ for $16 \mathrm{~h}$ in developing buffer $(1 \mathrm{M}$ Tris- $\mathrm{HCl}$, pH 7.5 with $\mathrm{CaCl}_{2}$ ). The gels were subsequently stained with $25 \%$ methanol $/ 8 \%$ acetic acid, containing Coomassie Brilliant Blue (Amresco, Solon, OH, USA). Gelatinase activity was visualized as white bands on a blue background, this represented the areas of proteolysis.

Mouse model of OVA-induced allergic asthma. Female $\mathrm{BALB} / \mathrm{c}$ specific pathogen-free mice (six weeks old) were purchased from Koatech Co. (Pyeongtaek, South Korea). Experimentation began after a two week quarantine and acclimatization period. All experimental procedures were approved by the Institutional Animal Care and Use Committee of the Korea Research Institute of Bioscience and Biotechnology. The mice were sensitized on days 0 and 14 , by an intraperitoneal (i.p) injection of $20 \mu \mathrm{g}$ OVA (Sigma-Aldrich, St. Louis, MO, USA) emulsified with $2 \mathrm{mg}$ aluminum hydroxide in $200 \mu 1$ phosphate-buffered saline (PBS) buffer (pH 7.4). On days 21,22 , and 23 , the mice received airway challenges of OVA $(1 \%(\mathrm{w} / \mathrm{v}))$ for $1 \mathrm{~h}$ using an ultrasonic nebulizer (NE-U12; Omron Corp., Tokyo, Japan). TO and montelukast, which was used as a positive control, were orally administered to the mice at a dose of $30 \mathrm{mg} / \mathrm{kg}$ body weight, one hour prior to OVA challenge. Airway responsiveness was indirectly assessed $24 \mathrm{~h}$ after the final challenge, using single-chamber, whole body plethysmography (Allmedicus Co. Ltd., Seoul, South Korea). The mice were sacrificed $48 \mathrm{~h}$ after the final challenge, by i.p injection of pentobarbital $(50 \mathrm{mg} / \mathrm{kg}$; Hanlim Pharm. Co. Ltd., Seoul, South Korea), and tracheostomies were performed. To obtain bronchoalveolar lavage fluid (BALF), ice-cold PBS $(0.5 \mathrm{ml})$ was infused into the lung three times, and the fluid was withdrawn each time by tracheal cannulation (total volume $1.5 \mathrm{ml}$ ). Total inflammatory cell numbers were determined by counting the cells in $\leq 5$ squares of a hemocytometer, following the exclusion of dead cells, with Trypan blue staining. Differential BALF cell counts were performed using Diff-Quik ${ }^{\circledR}$ staining reagent (IMEB Inc., San Marcos, CA, USA), according to the manufacturer's instructions.

Measurements of cytokine and chemokine levels in the BALF and IgE in the serum. The levels of IL-4, IL-5, and IL-13 in the BALF were measured using ELISA kits (R\&D SystemS, Minneapolis, MN, USA) according to the manufacturer's instructions. The levels of total IgE and OVA-specific IgE in the serum were also measured by ELISA. Microtiter plates were coated with anti-IgE antibodies (anti-mouse IgE; $10 \mathrm{~g} / \mathrm{ml}$; Serotec, Oxford, UK), in PBS-Tween ${ }^{\circledR} 20$, and incubated with BALF or a plasma sample. The plates were then washed four times with wash solution (PBS, containing $0.05 \%$ Tween 20 (Biosesang, GyeongGi-Do, Korea), and $200 \mu \mathrm{l}$ of o-Phenylenediamine dihydrochloride (Sigma-Aldrich) was added to each 
well. The plates were incubated for $10 \mathrm{~min}$ in the dark, and the absorbance was measured at $450 \mathrm{~nm}$.

Western blot analysis. The murine lung tissue was homogenized $(1 / 10 \mathrm{w} / \mathrm{v})$ using a homogenizer with Tissue Lysis/Extraction reagent (Sigma-Aldrich), containing a protease inhibitor cocktail (Sigma-Aldrich). Protein concentrations were determined using a protein assay reagent (Bio-Rad), according to the manufacturer's instructions. Equal amounts of total cellular protein $(30 \mu \mathrm{g})$ were separated by $12 \%$ SDS-PAGE and then transferred to polyvinylidene fluoride membranes (Millipore, Darmstadt, Germany). The membranes were incubated with blocking solution (5\% skim milk; Becton Dickinson, Franklin Lakes, NJ, USA), followed by an overnight incubation at $4^{\circ} \mathrm{C}$ with the appropriate primary antibody. The following primary antibodies and dilutions were used: anti- $\beta$-actin $(1: 2,000$ dilution; Cell Signaling Technology Inc., Danvers, MA, USA), anti-iNOS (1:1,000 dilution; Abcam, Cambridge, MA, USA) and anti-MMP-9 (1:1,000 dilution; Cell Signaling Technology Inc.). The blots were washed three times with Tris-buffered saline containing Tween ${ }^{\circledR} 20$ (TBST), followed by an incubation with a 1:10,000 dilution of horseradish peroxidase-conjugated secondary antibody (Jackson ImmunoResearch Laboratories Inc., Westgrove, PA, USA) for $30 \mathrm{~min}$ at room temperature. The blots were washed three additional times with TBST, and visualized using an enhanced chemiluminescence kit (Thermo Fisher Scientific, Waltham, MA, USA). Densitometic values for each protein were measured using a Chemi-Doc system (Bio-Rad).

Histology. After the BALF samples had been obtained, the lung tissues were fixed in $4 \%(\mathrm{v} / \mathrm{v})$ paraformaldehyde. The tissues were embedded in paraffin, sectioned at $4 \mu \mathrm{m}$, and stained with hematoxylin \& eosin solution (Sigma-Aldrich) and Periodic acid-Schiff (IMEB Inc) in order to estimate inflammation and mucus production, respectively.

Statistical analyses. The data are expressed as the means \pm standard error of the mean. Statistical significance was determined using analyses of variance, followed by multiple comparison tests with Dunnet's adjustment $\mathrm{A} P<0.05$ was considered to indicate a statistically significant difference.

\section{Results}

Effects of TO on NO production in LPS-stimulated RAW264.7 cells. Treatment with TO did not induce toxicity in RAW264.7 murine macrophage cells at concentrations $\leq 40 \mu \mathrm{g} / \mathrm{ml}$ (Fig. 1A). NO production was significantly increased in the LPS-stimulated RAW264.7 cells, as compared with the control cells. Conversely, the TO-treated cells exhibited concentration-dependent reductions in NO production, as compared with the LPS-stimulated RAW264.7 cells (Fig. 1B).

Effects of TO on the mRNA expressions levels of proinflammatory mediators in LPS-stimulated RAW264.7 cells. The LPS-stimulated RAW264.7 cells exhibited increased relative mRNA expression levels of iNOS, COX2, IL-6, TNF- $\alpha$ and MMP-9. However, TO-treated cells exhibited a significant reduction in the relative mRNA expression levels of these proinflammatory mediators, as compared with the LPS-stimulated
Table I. Serum levels of total immunoglobulin (Ig)-E and ovalbumin (OVA)-specific IgE in serum.

\begin{tabular}{lcc}
\hline Groups & $\begin{array}{c}\text { Total IgE } \\
(\mathrm{ng} / \mathrm{ml})\end{array}$ & $\begin{array}{c}\text { OVA-specific IgE } \\
(\mathrm{ng} / \mathrm{ml})\end{array}$ \\
\hline NC & $64.0 \pm 11.39$ & - \\
OVA & $1023.1 \pm 122.43^{\#}$ & $196.3 \pm 27.23^{\#}$ \\
Mon & $258.6 \pm 100.37^{*}$ & $67.2 \pm 23.0^{*}$ \\
TO & $453.9 \pm 110.53^{*}$ & $110.5 \pm 34.17^{*}$ \\
\hline
\end{tabular}

NC, normal control mice treated with phosphate-buffered saline only; OVA, OVA-sensitized/challenged mice; Mon, montelukast (30 mg/kg) plus OVA-sensitized/challenged mice; TO, Thuja orientalis $(30 \mathrm{mg} / \mathrm{kg}$ ) plus OVA-sensitized/challenged mice. ${ }^{~} \mathrm{P}<0.05$, as compared with $\mathrm{NC} ;{ }^{*} \mathrm{P}<0.05$, as compared with OVA.
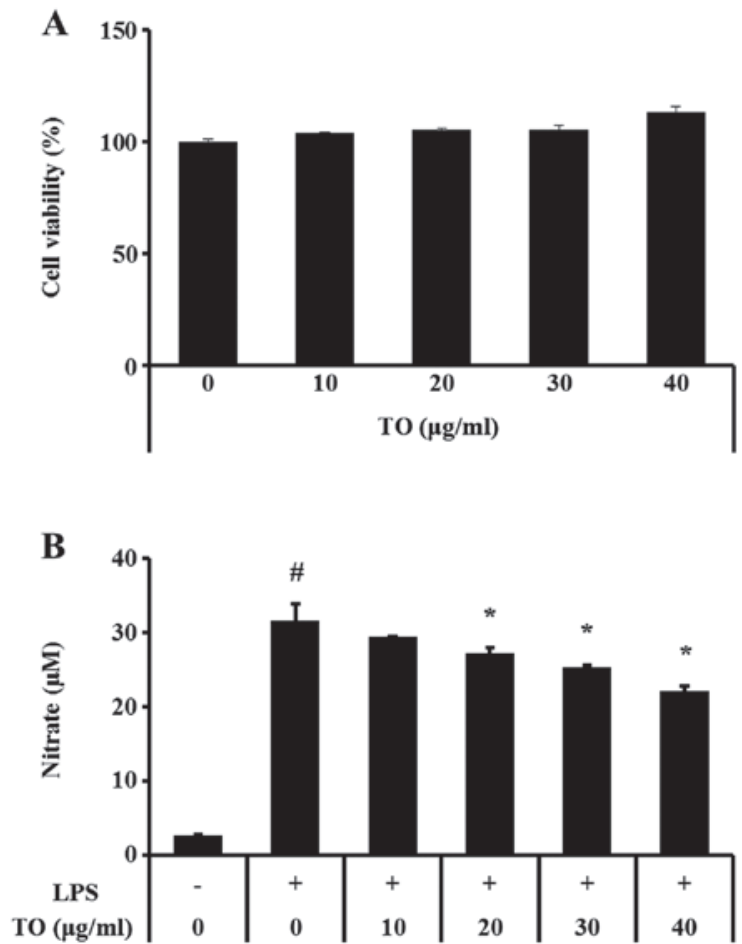

Figure 1. Thuja orientalis (TO) reduced the production of nitric oxide (NO) in RAW264.7 murine macrophage cells stimulated with lipopolysaccharide (LPS). The cells were treated with different concentrations $(10,20,30$, and $40 \mathrm{ng} / \mathrm{ml}$ ) of TO for $1 \mathrm{~h}$, and then incubated in the presence of LPS $(1 \mu \mathrm{g} / \mathrm{ml})$ for $24 \mathrm{~h}$. (A) Cell viability. (B) NO production. ${ }^{*} \mathrm{P}<0.05 \mathrm{vs}$. control; ${ }^{*} \mathrm{P}<0.05$ vs. cells treated with LPS alone.

cells (Fig. 2A). Furthermore, MMP-9 activity was increased in the LPS-stimulated RAW264.7 cells, whereas reductions were observed in the TO-treated cells (Fig. 2B).

Effects of TO on AHR in OVA-sensitized/challenged mice. AHR was significantly increased in the OVA-sensitized/challenged mice, as demonstrated by an increased concentration of methylcholine, as compared with the normal controls. The AHR, induced by the OVA challenge, was reduced in the positive control montelukast-treated mice. The TO-treated mice also exhibited significant reductions in AHR, as compared with the OVA-sensitized/challenged mice (Fig. 3). 
A

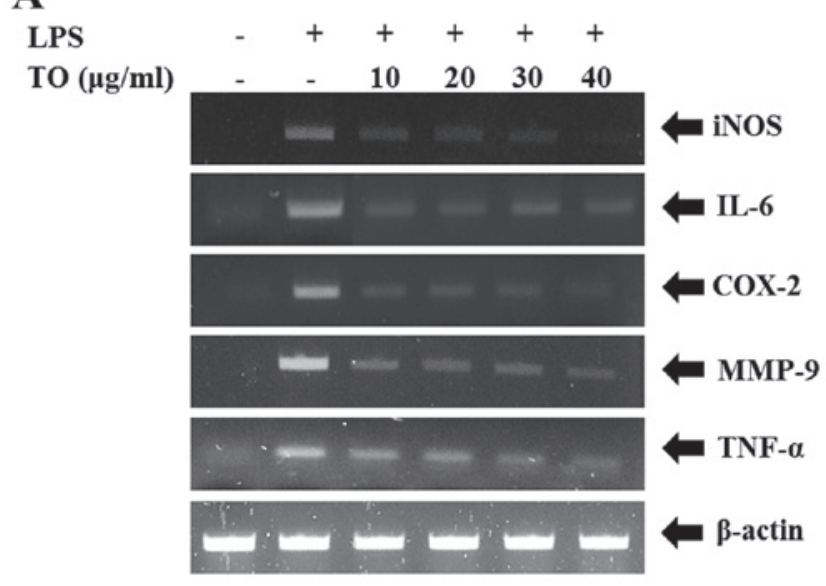

B

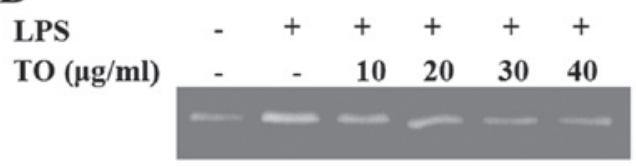

Figure 2. Thuja orientalis (TO) decreased the expression of proinflammatory mediators, including inducible nitric oxide synthase (iNOS), matrix metalloproteinase (MMP)-9, cylooxygenase (COX)-2, tumor necrosis factor (TNF)- $\alpha$ and interleukin (IL)-6 in RAW264.7 cells stimulated with lipopolysaccharide (LPS). The cells were treated with different concentrations (10, 20,30 , and $40 \mathrm{ng} / \mathrm{ml}$ ) of TO for $1 \mathrm{~h}$ and then incubated in the presence of LPS $(1 \mu \mathrm{g} / \mathrm{ml})$ for $24 \mathrm{~h}$ (A) Relative mRNA expression levels, as determined by reverse transcription polymerase chain reaction. (B) Gelatin zymography, to determine MMP-9 activity.

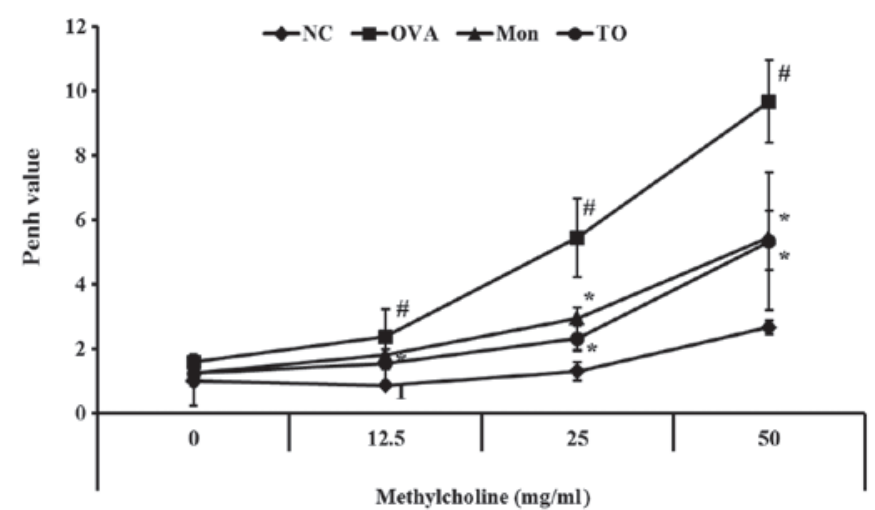

Figure 3. Thuja orientalis (TO) attenuated the airway hyperresponsiveness (AHR) induced by ovalubumin (OVA) challenge. Airway responsiveness was indirectly assessed $24 \mathrm{~h}$ after the final challenge using single-chamber, whole body plethysmography. OVA-sensitized/challenged mice exhibited significant increases in AHR with increasing methylcholine concentrations, as compared with the normal controls. However, the TO-treated mice exhibited marked reductions in AHR, as compared with the OVA-sensitized/challenged mice. $\mathrm{NC}$, normal control mice treated with phosphate-buffered saline only; OVA, OVA-sensitized/challenged mice; Mon, montelukast $(30 \mathrm{mg} / \mathrm{kg}$ ) plus OVA sensitized/challenged mice; TO, TO $(30 \mathrm{mg} / \mathrm{kg})$ plus OVA-sensitized/challenged mice. ${ }^{\#} \mathrm{P}<0.05$ vs. control mice; ${ }^{*} \mathrm{P}<0.05$ vs. OVA-sensitized/challenged mice.

Effects of TO on BALF inflammatory cell counts from OVA-sensitized/challenged mice. The OVA-sensitized/challenged mice exhibited a significant increase in the number of inflammatory cells present in the BALF, relative to the normal control mice (Fig. 4). However, the TO-treated mice exhibited

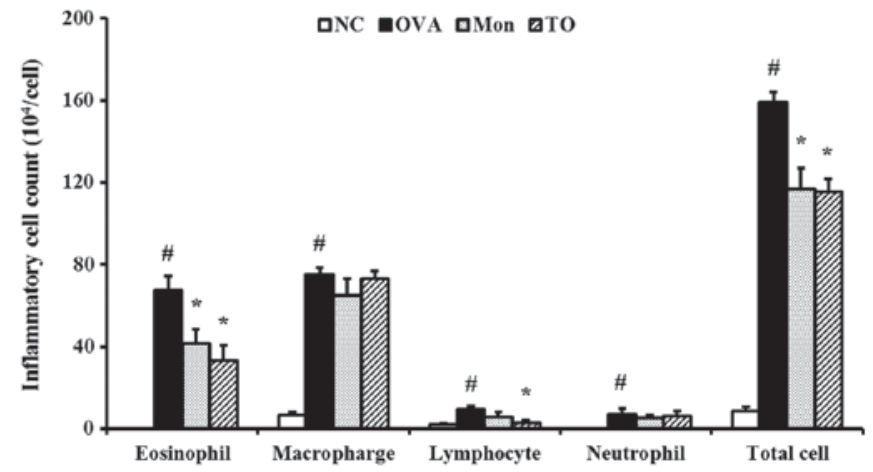

Figure 4. Thuja orientalis (TO) reduced the recruitment of inflammatory cells in the bronchial alveolar lavage fluid (BALF) induced by ovalbumin (OVA) challenge. The cells were isolated by centrifugation and stained with Diff-Quik ${ }^{\circledR}$ stain reagent. The cell numbers were determined using a light microscope with Trypan blue staining. The OVA-sensitized/challenged mice exhibited increased inflammatory cell counts in the BALF, relative to the normal control groups. Conversely, the TO-treated mice exhibited reduced inflammatory cell counts, particularly eosinophil counts, in the BALF, as compared with the OVA-sensitized/challenged mice. NC, normal control mice treated with phosphate buffered saline only; OVA, OVA-sensitized/challenged mice; Mon, montelukast $(30 \mathrm{mg} / \mathrm{kg}$ ) plus OVA-sensitized/challenged mice; TO, Thuja orientalis $(30 \mathrm{mg} / \mathrm{kg}$ ) plus OVA-sensitized/challenged mice

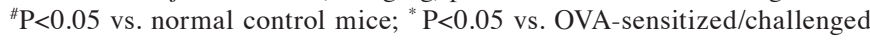
mice.

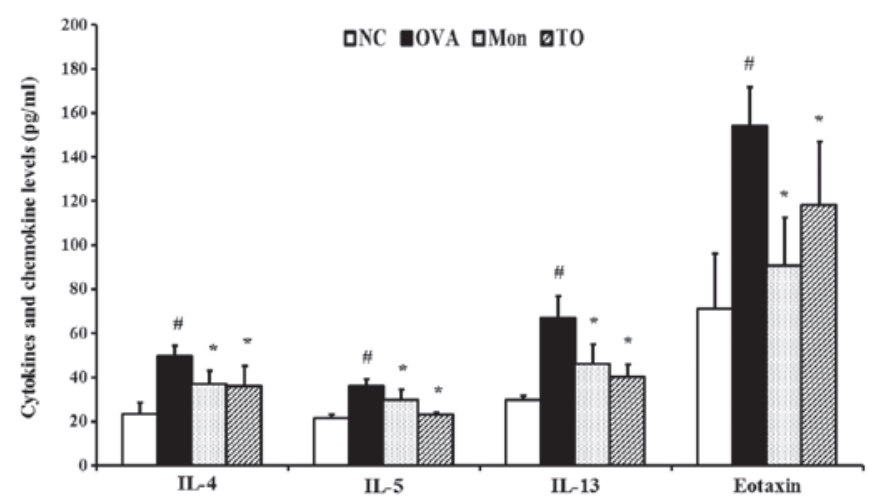

Figure 5. Thuja orientalis (TO) decreased the levels of $\mathrm{T}$ helper (Th)-2 cytokines and eotaxin in the bronchial alveolar lavage fluid (BALF). Th2 cytokines and eotaxin in the BALF were determined by ELISA. The ovalbumin (OVA)-sensitized/challenged mice exhibited increases in Th2 cytokines, including interleukin (IL)-4, IL-5, and IL-13 as compared with the normal controls. However, the TO-treated mice exhibited marked reductions in Th2 cytokines as compared with the OVA-sensitized/challenged mice. TO-treated mice also exhibited significant reductions in BALF eotaxin levels compared to the OVA-sensitized/challenged mice. NC, normal control mice treated with phosphate-buffered saline only; OVA, OVA-sensitized/challenged mice; Mon, montelukast (30 mg/kg) plus OVA-sensitized/challenged mice; TO, Thuja orientalis $\left(30 \mathrm{mg} / \mathrm{kg}\right.$ ) plus OVA-sensitized/challenged mice ${ }^{*} \mathrm{P}<0.05$ vs. normal control mice; " $\mathrm{P}<0.05$ vs. OVA-sensitized/challenged mice.

significant reductions in the number of inflammatory cells, particularly eosinophils, in the BALF, as compared with the OVA-sensitized/challenged mice.

Effects of TO on $I L-4, I L-5, I L-13$, and eotaxin in the BALF of OVA-sensitized/challenged mice. The OVA-sensitized/challenged mice showed marked elevations of Th2 cytokines, including IL-4, IL-5, and IL-13, in the BALF as compared with the normal controls. The elevations of Th2 cytokines 

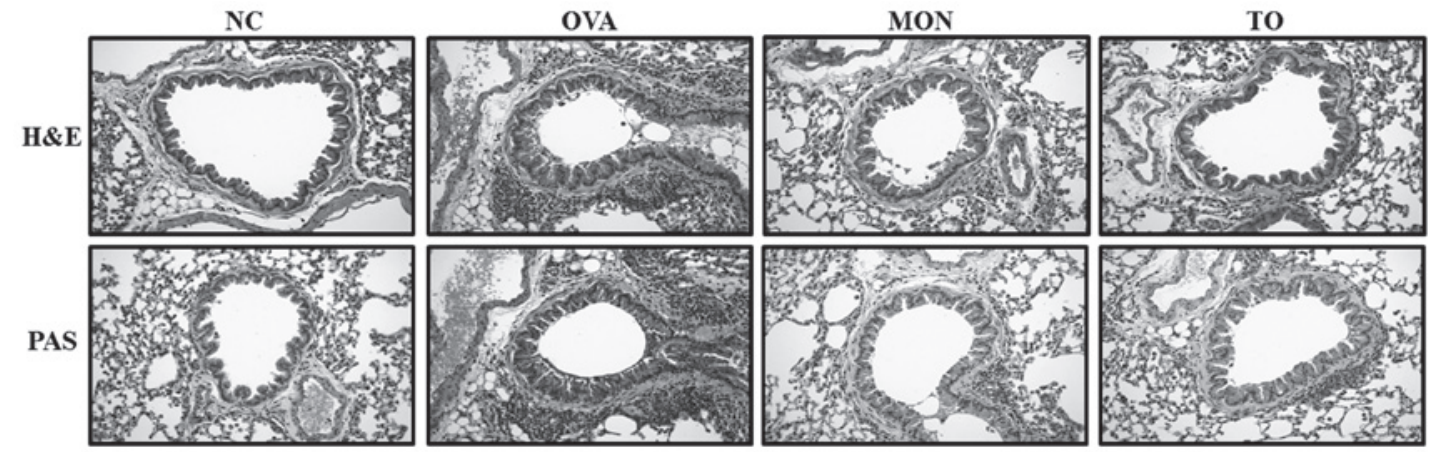

Figure 6. Thuja orientalis (TO) attenuated the airway inflammation and mucus production induced by ovalbumin (OVA) challenge, as determined by histological analysis (magnification, x200). The OVA-sensitized/challenged mice exhibited airway inflammation and mucus overproduction. However, the TO-treated mice exhibited reduced airway inflammation and mucus production as compared with the OVA-sensitized/challenged mice. NC, normal control mice treated with phosphate-buffered saline only; OVA, OVA-sensitized/challenged mice; Mon, montelukast ( $30 \mathrm{mg} / \mathrm{kg}$ ) plus OVA-sensitized/challenged mice; TO, Thuja orientalis $(30 \mathrm{mg} / \mathrm{kg}$ ) plus OVA-sensitized/challenged mice; H\&E, hematoxylin \& eosin; PAS, Periodic acid-Schiff.

induced by an OVA-challenge in the TO-treated mice were significantly reduced (Fig. 5). The production of eotaxin was also significantly increased in the OVA-sensitized/challenged mice; however, eotaxin production in the TO-treated mice was markedly reduced, as compared with the OVA-sensitized/challenged mice.

Effects of TO on total IgE and OVA-specific IgE in the serum of OVA-sensitized/challenged mice. Total IgE was markedly elevated in the OVA-sensitized/challenged mice, as compared with the normal controls (Table 1). However, the TO-treated mice exhibited a significant reduction in the total serum $\operatorname{IgE}$ levels, as compared with the OVA-sensitized/challenged mice. These findings were consistent with the OVA-specific IgE serum results. The OVA-sensitized/challenged mice exhibited significantly increased OVA-specific IgE levels in the serum, whereas the TO-treated mice exhibited marked reductions, as compared with the OVA-sensitized/challenged mice.

Effects of TO on inflammatory responses and mucus production in the lung tissue of OVA-challenged mice. Lung sections from the OVA-sensitized/challenged mice were examined for inflammatory cell infiltration into the peribronchial and perivascular lesions. The TO-treated mice exhibited reductions in airway inflammation, as compared with the OVA-sensitized/challenged mice (Fig. 6). Mucus production was also increased in the bronchial airways of the OVA-sensitized/challenged mice. Conversely, the TO-treated mice exhibited reductions in mucus production (Fig. 6).

Effects of TO on the expression of lung tissues of the OVA-sensitized/challenged mice. The relative protein expression levels of iNOS in the lung tissue was significantly increased in the OVA-sensitized/challenged mice, as compared with the normal controls (Fig. 7A and B). However, the TO-treated mice exhibited marked decreases in the protein expression of iNOS, as compared with the OVA-sensitized/challenged mice. The relative protein expression levels of MMP-9 in the lung tissue were similar to that of iNOS. The OVA-sensitized/challenged mice exhibited significantly increased MMP-9 protein expression in the lung tissue, as compared with the normal controls, whereas the TO-treated
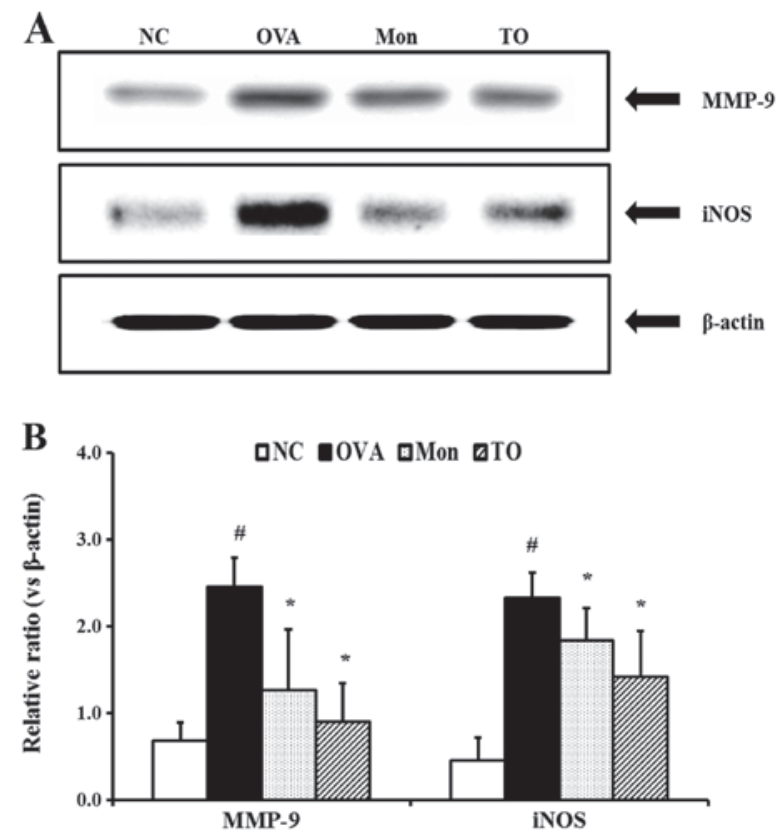

Figure 7. Thuja orientalis (TO) inhibited the relative expression levels of proinflammatory proteins in lung tissue induced by ovalbumin (OVA) challenge. (A) Relative protein expression of inducible nitric oxide synthase (iNOS) and matrix metalloproteinase (MMP)-9 in lung tissue, as determined by western blotting. (B) The relative ratios are expressed as the ratios of the densities of the proinflammatory mediators to $\beta$-actin. The OVA-sensitized/challenged mice exhibited significant increases in the expression of iNOS and MMP-9 as compared with the normal controls. The TO-treated mice exhibited marked reductions in the expression of these factors in lung tissue as compared with the OVA-sensitized/challenged mice. NC, normal control mice treated with phosphate-buffered saline only; OVA, OVA-sensitized/challenged mice; Mon, montelukast (30 mg/kg) plus OVA-sensitized/challenged mice; TO, Thuja orientalis $(30 \mathrm{mg} / \mathrm{kg})$ plus OVA-sensitized/challenged mice. \# $\mathrm{P}<0.05$ vs. control mice; ${ }^{*} \mathrm{P}<0.05$ vs. OVA-sensitized/challenged mice.

mice exhibited a marked decrease in the MMP-9 protein expression, relative to the OVA-sensitized/challenged mice.

\section{Discussion}

In the present study, the effects of TO on the development of asthma were evaluated using a murine model of 
OVA-induced asthma, and RAW264.7 murine macrophage cells. The TO-treated mice exhibited significant reductions in inflammatory cell numbers, Th2 cytokines and eotaxin in the BALF, serum IgE and AHR, as compared with the OVA sensitized/challenged mice. The OVA-challenge induced airway inflammation and mucus hypersecretion, which was shown to be suppressed in the lung tissues of the TO-treated mice; iNOS and MMP-9 expression levels were also reduced. In the LPS-stimulated RAW264.7 cells, TO treatment induced a significant reduction in NO production, and decreased the relative mRNA expression levels of iNOS, MMP-9, TNF- $\alpha$, COX-2 and IL-6.

Eosinophilic airway inflammation is an important feature of allergen-induced asthma. Eosinophils contain various toxic stimuli including cytotoxic proteins, lipid mediators, free radicals, and proinflammatory cytokines, which may cause asthmatic responses including AHR, mucus hypersecretion, and airway inflammation (16). The accumulation and activation of eosinophils has previously been shown to be associated with Th2 cytokines and chemokines $(17,18)$. Previous studies have demonstrated that Th2 cytokines, including IL-4, IL-5 and IL-13, can cause eosinophil development, activation and infiltration into the airways (19). Eotaxin is an eosinophil chemoattractant which induces the recruitment of eosinophils into asthmatic lesions (20). In the present study, OVA-sensitized/challenged mice exhibited significantly increased eosinophil BALF counts. However, the TO-treated mice exhibited marked reductions as compared with the OVA-sensitized/challenged mice; this finding was accompanied by reductions in Th2 cytokines and eotaxin. These results indicate that $\mathrm{TO}$ effectively suppressed eosinophilia induced by an OVA challenge. Furthermore, the findings of the histological analyses strongly supported the proposed effects of TO on asthma. In asthmatic conditions, Th2 cytokines cause the development and activation of eosinophils, which may aggravate asthmatic responses. Experimental asthma animal models exhibit inflammatory cell infiltration into peribronchail lesions, and mucus hypersecretion, both of which were exhibited by the OVA-sensitized/challenged mice in the present study. Conversely, the TO-treated mice exhibited attenuated airway inflammation and mucus production, as compared with the OVA-sensitized/challenged mice.

Proinflammatory mediators, including iNOS and MMP-9, have crucial roles in the development of asthma. NO is produced from L-arginine by iNOS, and acts as a strong activator of inflammatory signaling (21). Previous studies have shown that iNOS-derived NO aggravates airway inflammation in allergic asthma (22). Conversely, the suppression of iNOS attenuates asthmatic responses including airway constriction, inflammation and remodeling processes (7). Furthermore, the overexpression of iNOS results in an increase in MMP-9 which results in airway remodeling, via the breakdown of the lung tissue extracellular matrix (23). Previous studies have demonstrated that MMP-9 is associated with the production of numerous proinflammatory cytokines and growth factors, resulting in the infiltration of inflammatory cells into the airway $(24,25)$. In the present study, TO-treated mice exhibited significant reductions in relative MMP-9 and iNOS protein expression levels in lung tissue, as compared with the OVA-sensitized/challenged mice. These findings are consistent with the results of the in vitro experiment. TO treatment significantly reduced the production of NO, induced by LPS stimulation, in a concentration-dependent manner. TO treatment also effectively reduced the relative mRNA expression levels of MMP-9, iNOS, TNF- $\alpha$ and IL-6, in the LPS-stimulated RAW264.7 cells. These results indicate that the anti-asthmatic effects of TO are associated with the suppression of iNOS and MMP-9.

In conclusion, TO was shown to be capable of suppressing asthmatic responses, including eosinophilic airway inflammation, AHR and mucus hypersecretion in the OVA-sensitized/challenged mice; this may be through the inhibition of iNOS and MMP-9. Therefore, the present study suggests that TO may be used as a potential treatment for asthma.

\section{Acknowledgements}

The present study was supported by grants from the Korea Research Institute of Bioscience and Biotechnology Research Initiative Program of the Republic of Korea (no. KGM1221521).

\section{References}

1. Lee MY, Seo CS, Lee JA, et al: Anti-asthmatic effects of Angelica dahurica against ovalbumin-induced airway inflammation via upregulation of heme oxygenase-1. Food Chem Toxicol 49: 829-837, 2011 .

2. Gueders MM, Bertholet P, Perin F, et al: A novel formulation of inhaled doxycycline reduces allergen-induced inflammation, hyperresponsiveness and remodeling by matrix metalloproteinases and cytokines modulation in a mouse model of asthma. Biochem Pharmacol 75: 514-526, 2008.

3. Holgate ST: The airway epithelium is central to the pathogenesis of asthma. Allergol Int 57: 1-10, 2008.

4. Zhou DY, Du Q, Li RR, Huang M,Zhang Q and Wei GZ: Grape seed proanthocyanidin extract attenuates airway inflammation and hyperresponsiveness in a murine model of asthma by downregulating inducible nitric oxide synthase. Planta Med 77: 1575-1581, 2011.

5. Ricciardolo FL, Sterk PJ, Gaston B and Folkerts G: Nitric oxide in health and disease of the respiratory system. Physiol Rev 84: 731-765, 2004.

6. Islam T, Breton C, Salam MT, et al: Role of inducible nitric oxide synthase in asthma risk and lung function growth during adolescence. Thorax 65: 139-145, 2010.

7. Prado CM, Leick-Maldonado EA, Yano L, et al: Effects of nitric oxide synthases in chronic allergic airway inflammation and remodeling. Am J Respir Cell Mol Biol 35: 457-465, 2006.

8. Jung SH, Kim BJ, Lee EH and Osborne NN: Isoquercitrin is the most effective antioxidant in the plant Thuja orientalis and able to counteract oxidative-induced damage to a transformed cell line (RGC-5 cells). Neurochem Int 57: 713-721, 2010.

9. Lee YJ, Hwang SM, Yoon JJ, et al: Inhibitory effect of Thuja orientalis on TNF- $\alpha$-induced vascular inflammation. Phytother Res 24: 1489-1495, 2010.

10. Chae HS and Chin YW: Anti-allergic effects of lambertianic acid from Thuja orientalis in mouse bone marrow-derived mast cells. Immunopharmacol Immunotoxicol 34: 250-255, 2012.

11. Won JN, Lee SY, Song DS and Poo H: Antiviral activity of the plant extracts from Thuja orientalis, Aster spathulifolius, and Pinus thunbergii against influenza virus A/PR/8/34. J Microbiol Biotechnol 23: $125-130,2013$

12. Xu GH, Ryoo IJ, Kim YH, Choo SJ and Yoo ID: Free radical scavenging and antielastase activities of flavonoids from the fruits of Thuja orientalis. Arch Pharm Res 32: 275-282, 2009.

13. Jung HW, Kang SY, Park KH, et al: Effect of the semen extract of Thuja orientalis on inflammatory responses in transient focal cerebral ischemia rat model and LPS-stimulated BV-2 microglia. Am J Chin Med 41: 99-117, 2013.

14. Kim TH, Li H, Wu Q, Lee HJ and Ryu JH: A new labdane diterpenoide with anti-inflammatory activity from Thuja orientalis. J Ethnopharmacol 146: 760-767, 2013. 
15. Heussen C and Dowdel EB: Electrophoretic analysis of plasminogen activators in polyacrylamide gels containing sodium dodecyl sulfate and copolymerized substrate. Anal Biochem 102: 196-202, 1980.

16. Uhm TG, Kim BS and Chung IY: Eosinophil development, regulation of eosinophil-specific genes, and role of eosinophils in the pathogenesis of asthma. Allergy Asthma Immunol Res 4: 68-79, 2012.

17. Pope SM, Brandt EB, Mishra A, et al: IL-13 induces eosinophil recruitment into the lung by an IL-5- and eotaxin-dependent mechanism. J Allergy Clin Immunol 108: 594-601, 2001.

18. Pope SM, Zimmermann N, Stringer KF, Karow ML and Rothenberg ME: The eotaxin chemokines and CCR3 are fundamental regulators of allergen-induced pulmonary eosinophilia. J Immuno 175: 5341-5350, 2005.

19. Mould AW, Ramsay AJ, Matthaei KI, Young IG, Rothernberg ME and Foster PS: The effects of IL-5 and eotaxin expression in the lung on eosinophil trafficking and degranulation and the induction of bronchial hyperreactivity. J Immunol 164: 2142-2150, 2000.
20. Pease JE and Williams TJ. Chemokines and their receptors in allergic disease. J Allergy Clin Immunol 118: 305-318, 2006.

21. Hesslinger C, Strub A, Boer R, Ulrich WR, Lehner MD and Braun C: Inhibition of inducible nitric oxide synthase in respiratory diseases. Biochem Soc Trans 37: 886-891, 2009.

22. Starling CM, Prado CM, Leick-Maldonado EA, et al: Inducible nitric oxide synthase inhibition attenuates lung tissue responsiveness and remodeling in a model of chronic pulmonary inflammation in guinea pigs. Respir Physiol Neurobiol 165: 185-194, 2009.

23. Prado CM, Yano L, Rocha G, et al: Effects of inducible nitric oxide synthase inhibition in bronchial vascular remodeling-induced by chronic allergic pulmonary inflammation. Exp Lung Res 37: 259-268, 2011

24. Vermaelen KY, Cataldo D, Tournoy K, et al: Matrix metalloproteinase-9-mediated dendritic cell recruitment into the airways is a critical step in a mouse model of asthma. J Immunol 171: 1016-1022, 2003.

25. McMillan SJ, Kearley J, Campbell JD, et al: Matrix metalloproteinase- 9 deficiency results in enhanced allergen-induced airway inflammation. J Immunol 172: 2586-2594, 2004. 